\title{
Simple numerical simulation of Strain Measurement
}

\author{
H. Tai \\ Langley Research Center, NASA, Hampton VA 23681
}

\begin{abstract}
By adopting the basic principle of the reflection (and transmission) of a plane polarized electromagnetic wave incident normal to a stack of films of alternating refractive index, a simple numerical code was written to simulate the maximum reflectivity (transmittivity) of a fiber optic Bragg grating corresponding to various non- uniform strain conditions including photo-elastic effect in certain cases.
\end{abstract}

Keywords: Bragg grating, reflectivity, non-uniform strain, photo-elastic effect

\section{INTRODUCTION}

Among many uses as a sensing device, the optical fiber Bragg gratings have been used widely as a tool for measuring strain in structural testing because of its lightweight and portability. In the simplest form a fiber Bragg grating consists of a periodic modulation of the refractive index in a core of a single mode optical fiber, where the phase fronts are perpendicular to the fiber's longitudinal axis and with grating planes having constant period. Light, guided along the core of an optical fiber, will be seattered by each grating plane. If the Bragg condition is not satisfied, the reflected light from each of the subsequent planes becomes progressively out of phase and will eventually cancel out. If the Bragg condition is satisfied, the contributions of reflected light from each grating plane add constructively in the backward direction to form a back reflected peak with center wavelength defined by the grating period. As the grating period is changed in a mechanical strain test, the center peak of the reflected wavelength correspondingly changes to a new location, and the strain can be deduced. However, when the strain is large, the local fiber index of refraction is changed due to the photo-elastic effect which in turn affects the reflected spectrum. This is the underlying principle behind this measurement technology. Since typically, the grating has physical dimension of $1 \sim 5 \mathrm{~mm}$ length, the deformation may not be uniformly distributed across the whole length of the grating. In the extreme case whereby the deformation (or strain) is totally randomly distributed and large, then there is negligible light reflected at any wavelength. The present work intends to investigate the behavior in between the ideal Bragg condition and the negligible reflected light condition, i.e. a well behaved non-uniform periodicity condition. In other words, if we modulate the grating period in a controlled fashion, we can numerically simulate the reflected spectrum and conversely, once we have the reflected spectrum, we would be able to invert the problem and gain some understanding that would be highly useful for non-uniform strain measurement.

\section{THEORETICAL BACKGROUND}

For the last thirty years, a tremendous number of technical articles have been written on periodic dielectric wave-guides and a few representative ones are listed here ${ }^{1-15}$. On the theoretical side, the most common methods fell into either the matrix method, or the coupled-wave method. The use of the matrix method in the study of the propagation of plane electromagnetic waves (TEM) through a stratified medium is well documented in optics ${ }^{16,17}$. It is justified to assume a TEM wave in a common optical fiber under the weakly guiding condition ${ }^{18}$. The coupled-wave method has long been used to analyze the characteristics for a periodic layered medium because it gives simple analytic expressions. Ref. 13 showed that these two methods gave identical results. Ref. 15 asserted that the coupled mode theory is derived as an approximation for weak periodic structures. However, we feel that the matrix method has the ability to interrogate the grating in a more detailed fashion, thus presenting a better physical picture for describing the relationship between grating structure and reflection spectrum. For uniform strain, it is known that a compact expression can be readily obtained for the reflected spectrum as a function of grating distance ${ }^{16}$. The matrix method is outlined below following Ref. 17 with minor change of notation. A linear y polarized electromagnetic wave propagates at normal incidence on stack of films of individual thickness $d_{i}\left(d_{1}=0\right)$ 
having refractive index $n_{i}$. We assumed the dielectrics films to be non-absorbing. The electric fields in the ith layer can be represented as (omitting the part $e^{-i \omega t}$ )

$\overrightarrow{\mathbf{E}_{i}}=\hat{y} E_{i}^{+} e^{+i k_{i}\left(x-\sum_{j=1}^{i-1} d_{j}\right)}+\hat{y} E_{i}^{-} e^{-i k_{i}\left(x-\sum_{j=1}^{i-1} d_{j}\right)}$

where $\hat{y}$ represents a unit vector in the $y$ direction and the quantity $E_{i}^{+} e^{i k_{i}(x-\ldots)}$ represents a plane wave propagating in the positive $\mathrm{x}$ direction with an amplitude $E_{i}^{+}$; the quantity $E_{i}^{-} e^{-i k_{i}(x-\ldots)}$ represents a plane wave propagating in the negative x direction with an amplitude $E_{i}^{-}$etc.

$k_{i}=(\omega / c) n_{i}=k_{0} n_{i}$

$n_{i}$ and $k_{i}$ are the refractive index and propagation constant (wave number) respectively in region $i$ and $k_{0}=\omega / c=2 \pi / \lambda$ represents the free space wave number. The corresponding magnetic fields $\overrightarrow{\mathbf{H}}$ can be evaluated as

$$
\overrightarrow{\mathbf{H}}=\vec{k} \times \overrightarrow{\mathbf{E}} / \omega \mu
$$

$$
\overline{\mathbf{H}_{i}}=\hat{z} \frac{n_{i}}{c \mu_{0}} E_{i}^{+} e^{i k_{i}\left(x-\sum_{j=1}^{i-1} d_{j}\right)}-\hat{z} \frac{n_{i}}{c \mu_{0}} E_{i}^{-} e^{-i k_{i}\left(x-\sum_{j=1}^{i-1} d_{j}\right)}
$$

where the media are assumed to be nonmagnetic with $\mu=\mu_{0}$. By imposing the condition that the tangential components of fields $\overrightarrow{\mathbf{E}}$ and $\overrightarrow{\mathbf{H}}$ be continuous at the interface separating $i t h$ and $i+1$ th layers, we can write

$$
\left(\begin{array}{c}
E_{i}^{+} \\
E_{i}^{-}
\end{array}\right)=\frac{1}{t_{i}}\left(\begin{array}{cc}
e^{-i \delta_{i}} & r_{i} e^{-i \delta_{i}} \\
r_{i} e^{i \delta_{i}} & e^{i \delta_{i}}
\end{array}\right)\left(\begin{array}{c}
E_{i+1}^{+} \\
E_{i+1}^{-}
\end{array}\right)=S_{i}\left(\begin{array}{c}
E_{i+1}^{+} \\
E_{i+1}^{-}
\end{array}\right)
$$

where $r_{i}$ and $t_{i}$ are the amplitude of reflection and transmission coefficients at the $i t h$ interface and are given by

$$
r_{i}=\frac{n_{i}-n_{i+1}}{n_{i}+n_{i+1}}, \quad t_{i}=\frac{2 n_{i}}{n_{i}+n_{i+1}}
$$

and $S_{i}$ is referred to as the transfer function at the $i t h$ interface.

And $\delta_{i}=k_{i} d_{i}=n_{i} k_{0} d_{i}$

Generalizing the analysis for $N$ films and for normal incidence, we have

$$
\left(\begin{array}{c}
E_{1}^{+} \\
E_{1}^{-}
\end{array}\right)=S\left(\begin{array}{c}
E_{N+2}^{+} \\
0
\end{array}\right)
$$

where $S=s_{1} s_{2} \ldots S_{N+1}$

$\delta_{1}=0, \delta_{j}=k_{j} d_{j}=2 \pi n_{j} d_{j} / \lambda_{0} ; j=2,3, \ldots N+1$

Therefore the amplitude of reflection and transmission coefficients of the $N$ films are given by

$$
r=E_{1}^{-} / E_{1}^{+} \text {and } t=E_{N+2}^{+} / E_{1}^{+}
$$


The reflectivity and transmittivity are therefore given by

$$
R=|r|^{2} \text { and } T=|t|^{2}
$$

The matrix method mentioned above is applied here to calculate the reflectivity and transmissivity of a periodic medium or near periodic medium, i.e., for the case of Bragg grating. The Bragg grating is represented by a stack of layers with equal thickness $d / 2$ and with alternating refractive indices

$(n+\Delta n)$ and $n$. The wavelength corresponding to maximum reflectivity corresponds to a free space wavelength, $\lambda$ and is given by

$\lambda=2\left(n+\frac{\Delta n}{2}\right) d$

where $d$ represents the spatial periodicity of the index of refraction. and $\left(n+\frac{\Delta n}{2}\right)$ is the average index of refraction of each period.

\section{SIMULATION PROCEDURE AND RESULTS}

Based on realistic strain measurements, the following set of parameters are chosen; $n=1.45, \Delta n=10^{-3}$, the alternating layers having index of $(n+\Delta n)$ and $n$, scanning laser wavelength $\lambda$ $=1530 \sim 1590 \mathrm{~nm}$, grating parameter $d=\lambda 2(n+\Delta n / 2)$. With the information mentioned above and a grating of typical length of $1 \sim 5 \mathrm{~mm}$, the number of layers in the grating is in the neighborhood of thousands. The simulation done here uses 1500 periods (3000 layers). The strain normally is in the range of $10^{-2}$ to $10^{-4}$ or $10,000 \sim 100 \mu \varepsilon$, that corresponds to a peak wavelength shift within $15 \mathrm{~nm}$, provided the strain is uniformly distributed. In a realistic situation the strain may not be totally uniform and therefore we can assume some simple continuous modulation functions to mimic the grating spacing as a function of grating distance. In other words the calculation is performed numerically grating plane by grating plane with each grating plane distance changed in a controlled fashion. The simple modulation functions chosen are listed below.

\section{3a. Uniform Strain}

If the unstrained grating period is denoted as $d$, under strained condition the period changed to $d$ ' as

$$
d^{\prime}=d+d \varepsilon
$$

where $\varepsilon$ is the strain. For $\varepsilon=10^{-3}, 10^{-2}$, and $2 \times 10^{-2}$ the reflected spectra are plotted in Fig. 1,2 and 3 . Of course $\varepsilon$ here can be negative which means the deformation is due to compression rather than elongation.

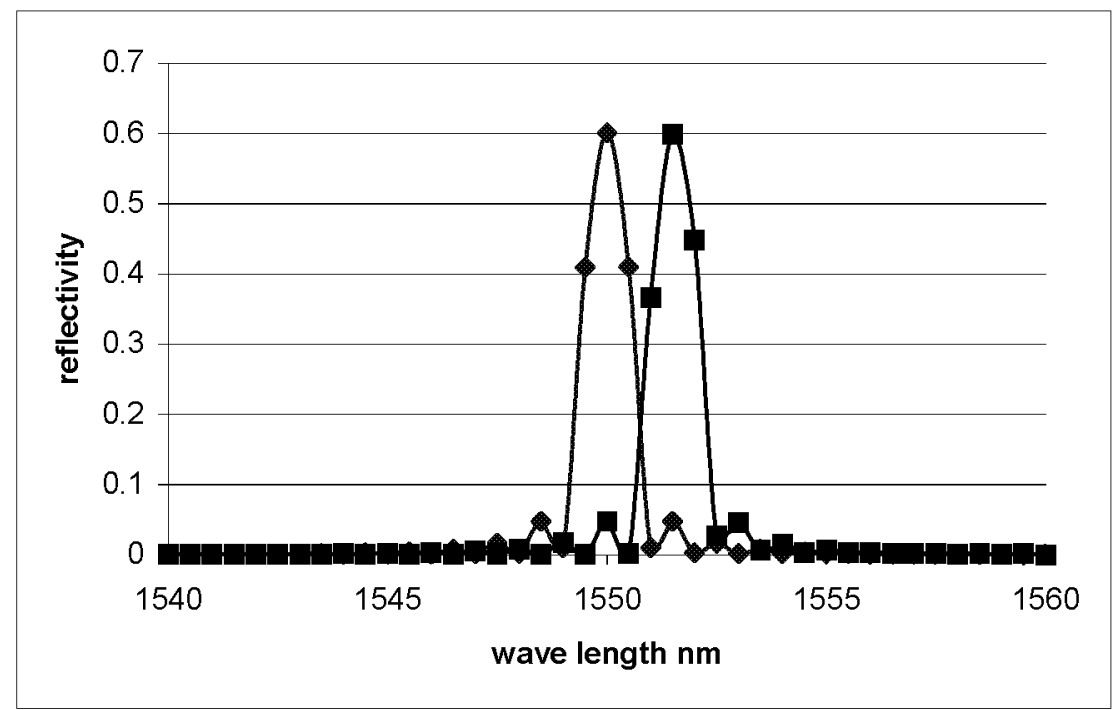

Fig. 1 The spectrum curve for uniform strain $\varepsilon=10^{-3}$ is on the right. The curve on the left is the unstrained spectrum 


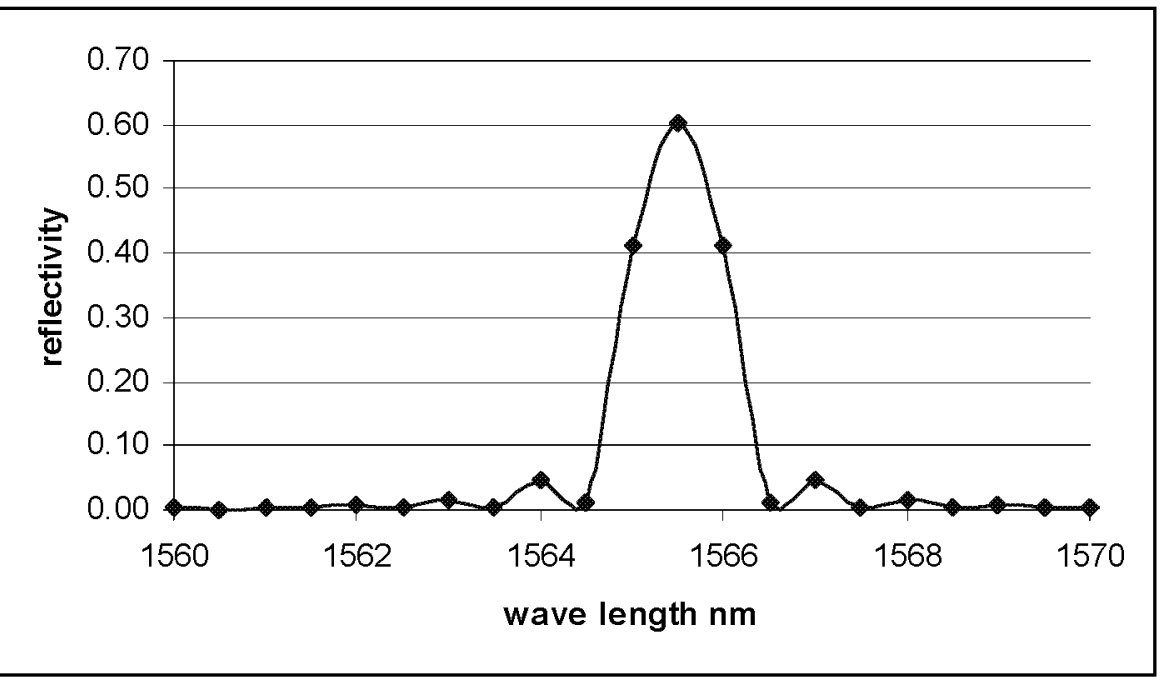

Fig. 2 uniform $\operatorname{strain} \varepsilon=10^{-2}$

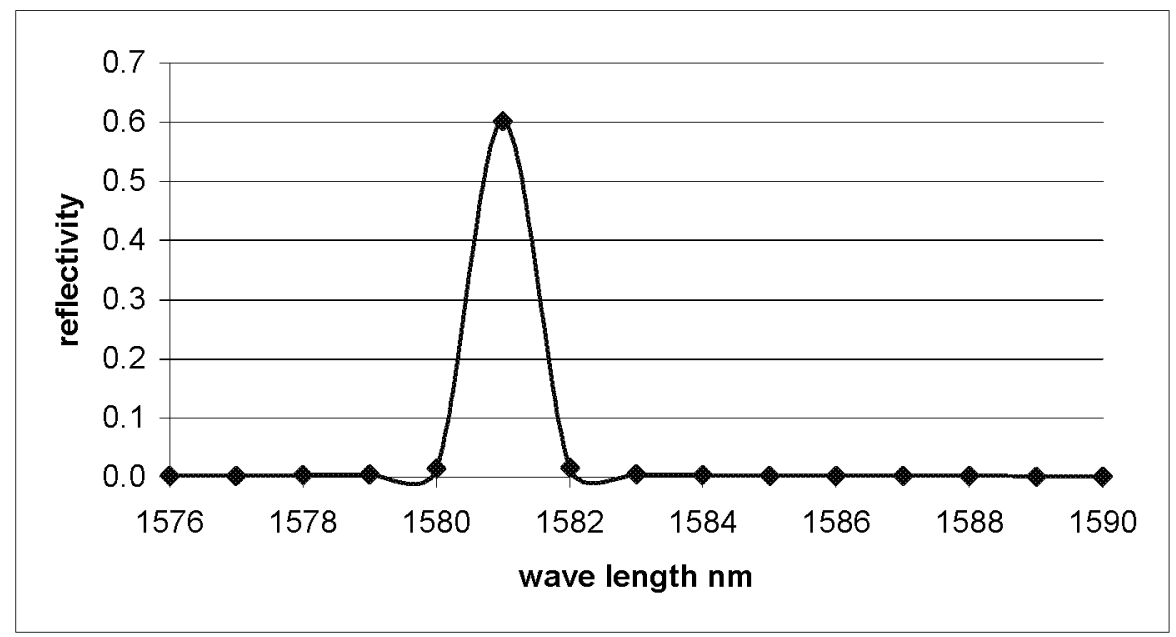

Fig. 3 uniform strain $\varepsilon=2 \times 10^{-2}$

For example, in Fig.2 under strain the reflected peak is located at $1565.5 \mathrm{~nm}$ which corresponds to a wavelength shift due to strain with strained spacing of $d$ '

$d^{\prime}=\frac{1565.5}{2 \times 1.4505}=539.6415029 \mathrm{~nm}$

$\varepsilon=\frac{d^{\prime}-d}{d}=\frac{539.6415029-534.2985178}{534.2985178} \simeq \frac{5.3429851}{534.29851}=10^{-2}$.

This verifies the basic principle of Bragg grating technology for strain measurement.

\section{B. Linear Strain}


We assume the strain is a linear function of grating distance $\mathrm{x}$ and $\varepsilon$ is the maximum strain which occurs at the farther end of the grating, at the of length $\mathrm{x}=L$.

$$
d^{\prime}(x)=d+d \frac{x}{L} \varepsilon
$$

Because the symmetry associated with the system, we can not differentiate this set up from the set up where the maximum strain at the beginning of the grating, i.e.,

$$
d^{\prime}(x)=d+d\left(1-\frac{x}{L}\right) \varepsilon
$$

the reflected light spectrum should be identical. The spectra are plotted in Figs. 4, 5 and 6.

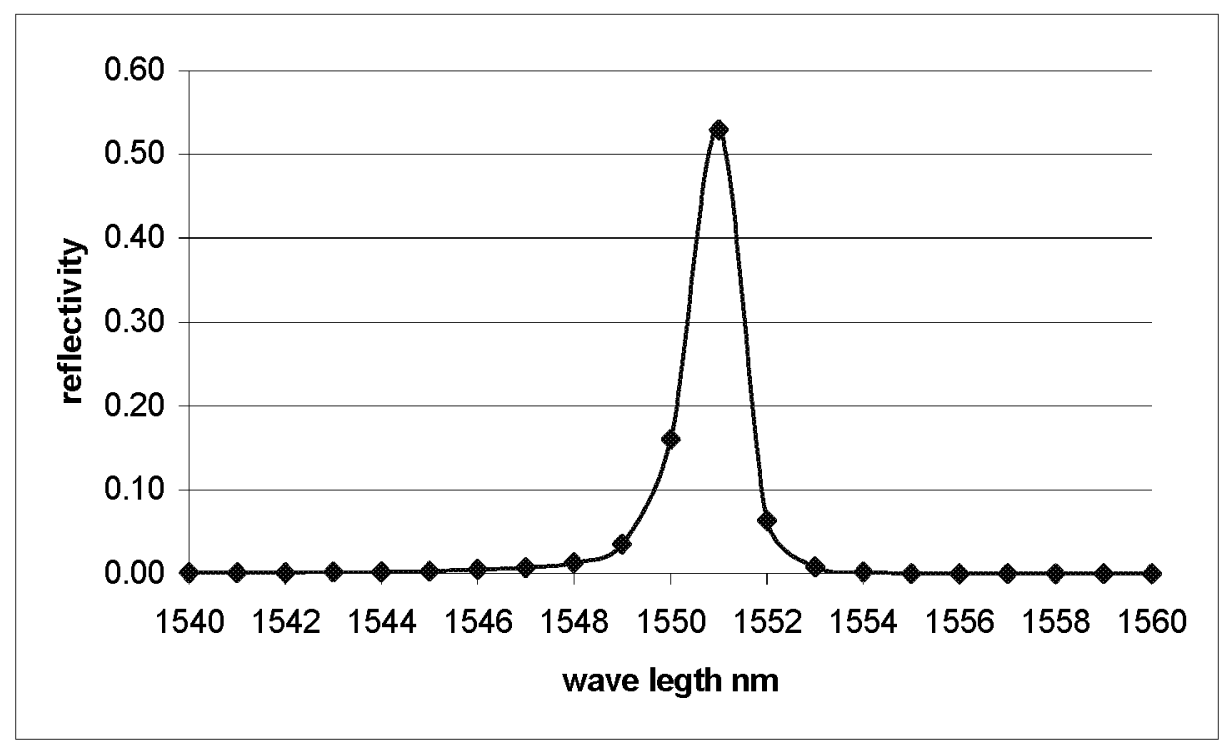

Fig. 4 linear strain where the maximum strain is at the end of grating $\varepsilon=10^{-3}$

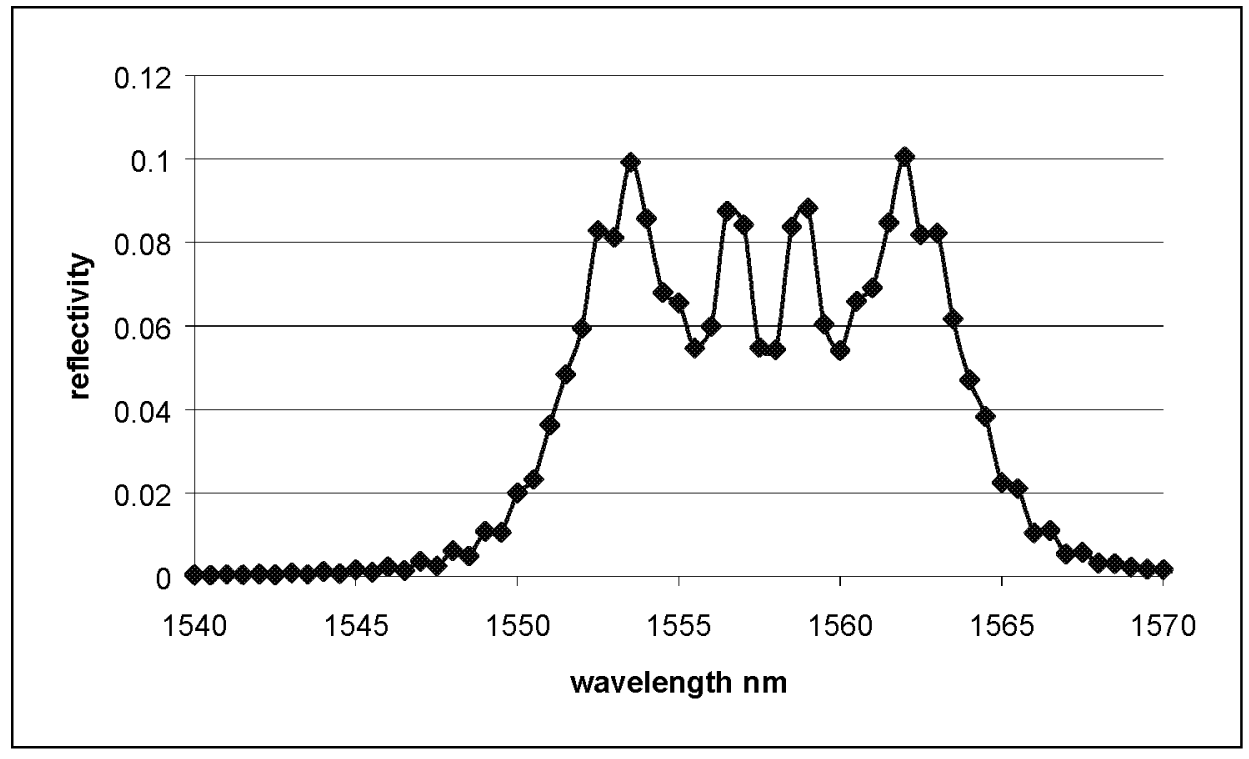

Fig. 5 linear strain where the maximum strain is at the end of grating, $\varepsilon=10^{-2}$ 


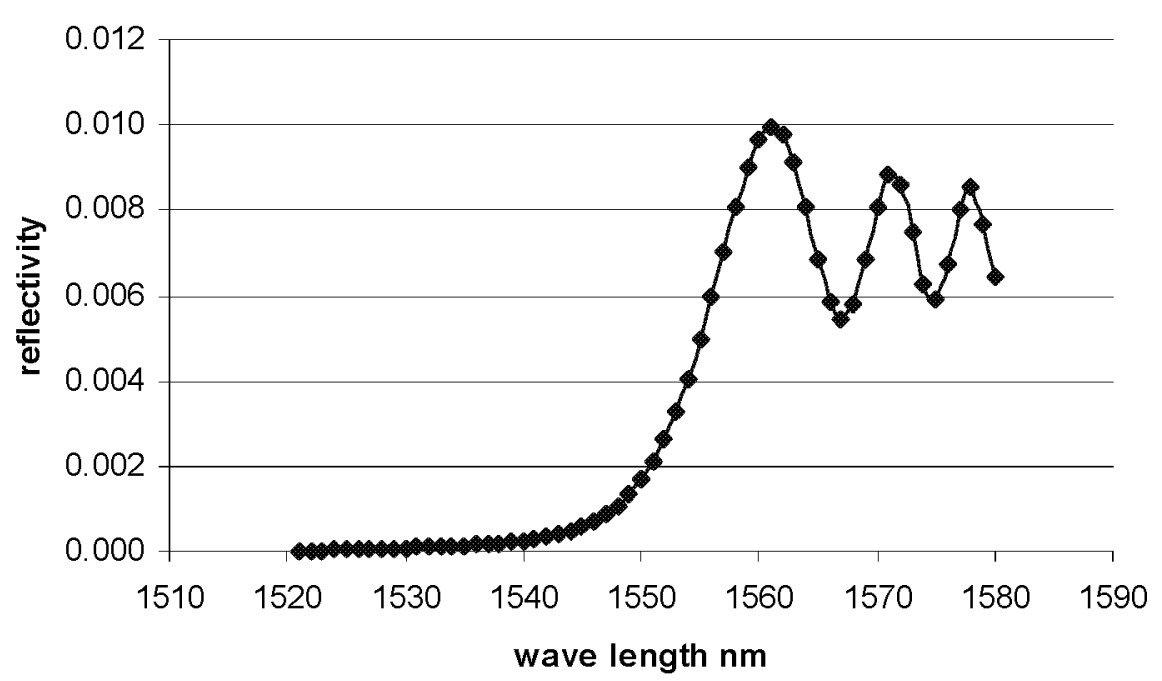

Fig. 6 linear strain where the maximum strain is at the end of grating, $\varepsilon=10^{-1}$

It is quite likely that in a static measurement the strain may have a linear distribution, especially if the Bragg grating covers a larger distance, e.g., $5 \mathrm{~mm}$. However if the strain is small, a sharp peak is still shown. If the strain increases to the order of $10^{-2}$, we have a totally different picture; multiple peaks appear in the spectrum. Also the height of the peaks greatly reduced. It is customary to use geometrical means, such as weighted average to assign a strain. From Fig. 5 and Fig. 2 of uniform strain we can verify that indeed the mean value of the wavelength in Fig. 5 predicts the correct strain since half of the maximum strain in the linear case is the strain value of the uniform case. If the strain further increases, the peaks of the spectrum further reduced and the spectrum tends to get broader. This kind of shape of spectrum usually is termed as chirped spectrum ${ }^{19}$.

\section{3c. The sinusoidal strain distribution}

We assume the strain has a sinusoidal behavior where the maximum strain occurs at the middle point of the grating, so the sine function has a period of twice of grating length.

$$
d^{\prime}(x)=d+d \varepsilon \sin \frac{\pi x}{L}
$$

This simulates in the expansion experiments, the middle section stretches the most. If $\varepsilon$ is negative implying the middle section of the grating suffers the greatest compression strain. Again the reflected spectra are shown in Figs.7, 8 and 9. 


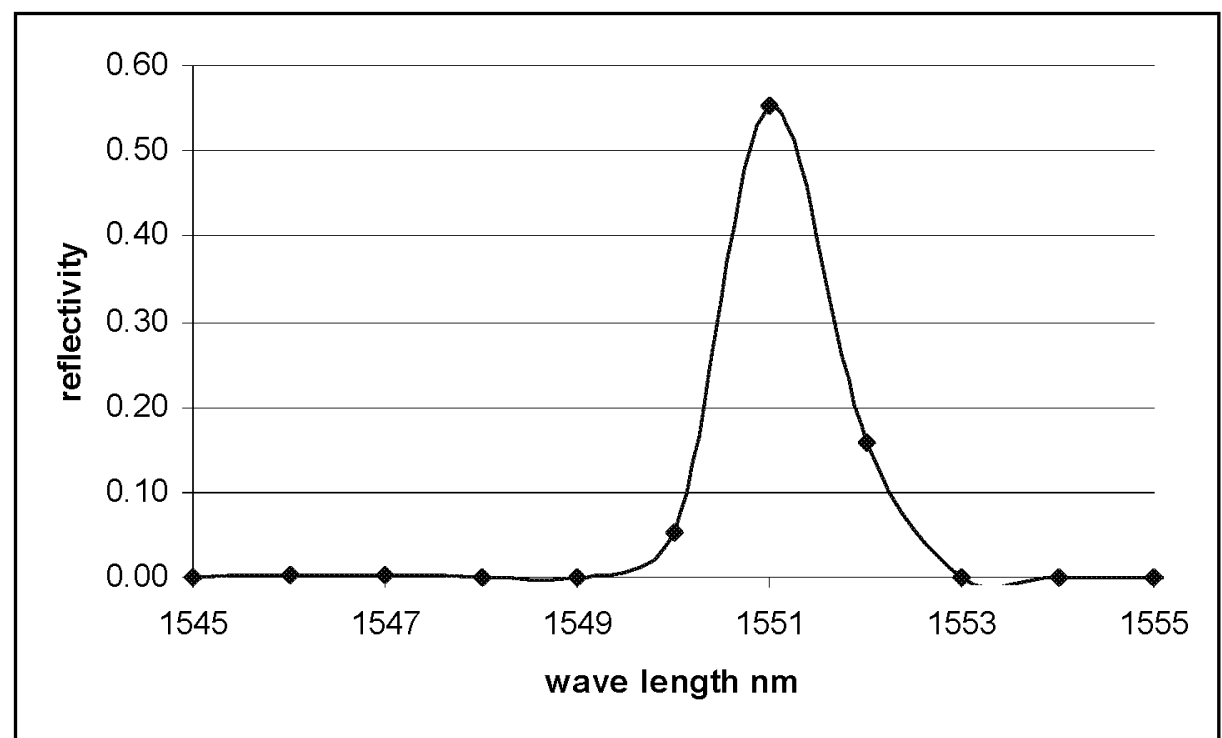

Fig. 7 strain has a sine curve distribution where the middle section has the greatest strain of $10^{-3}$

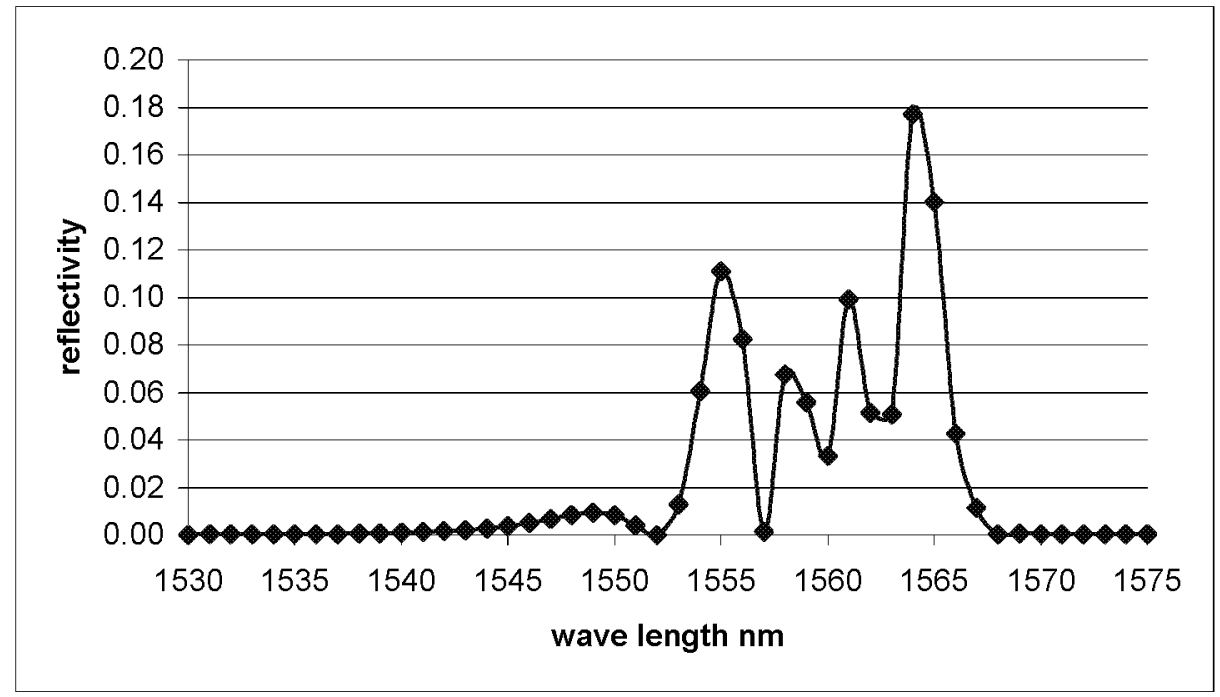

Fig. 8 strain has a sine curve distribution where the middle section has the greatest strain of $10^{-2}$ 


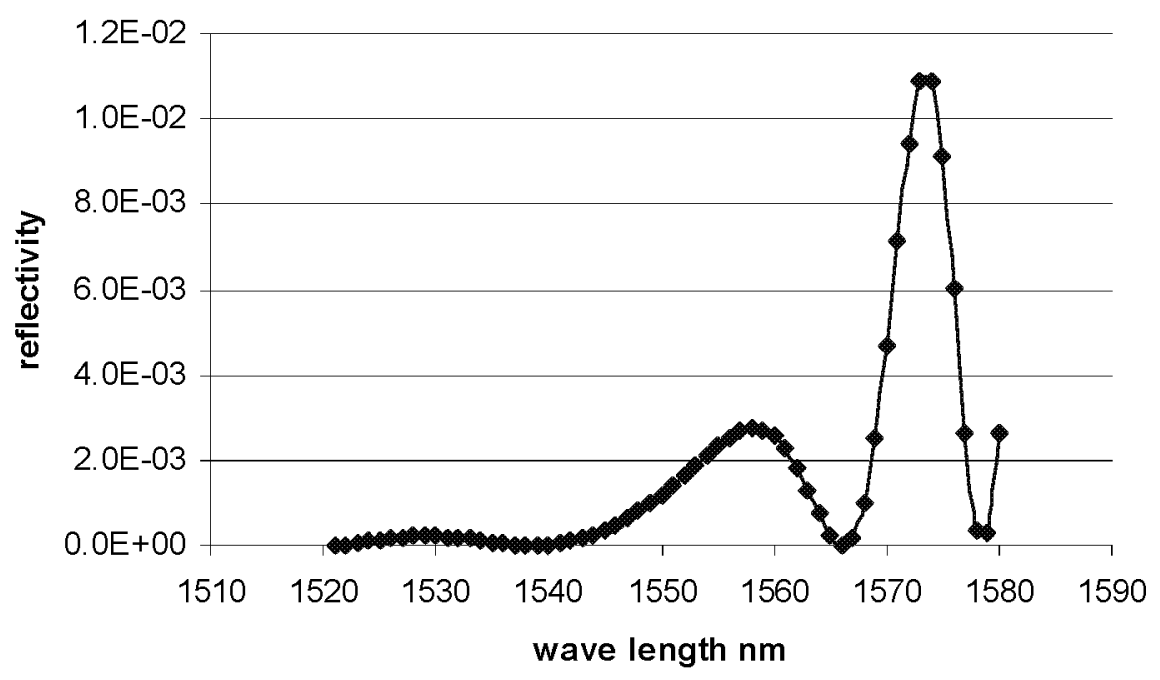

Fig. 9 strain has a sine curve distribution where the middle section has the greatest strain of $10^{-1}$

As in the linear strain case, the sine curve distribution also presents similar spectrum. Where the strain is small $\left(10^{-3}\right)$, we still see a sharp peak. However if the strain is increased, multiple peaks begin to show up and magnitude of the reflected signal is greatly reduced.

\section{3d. Random strain distribution}

We can easily have a random strain distribution by invoking a constant strain multiplied by a random number, rand having a value between 0 and 1 .

$d^{\prime}=d+d \varepsilon$ rand

The reflected spectra for the random case are shown in Figs. (10) and (11)

Again when the strain is small we are still able to see a sharp peak even at $\varepsilon=10^{-2}$. However, when the strain reaches to $10^{-1}$, we see (or don't see) that the spectrum is totally changed. Since the intensity is so small, the grating response is unrecognizable and in the real world would be mistaken for signal noise. This verifies our belief that based on the fundamental physics principle.

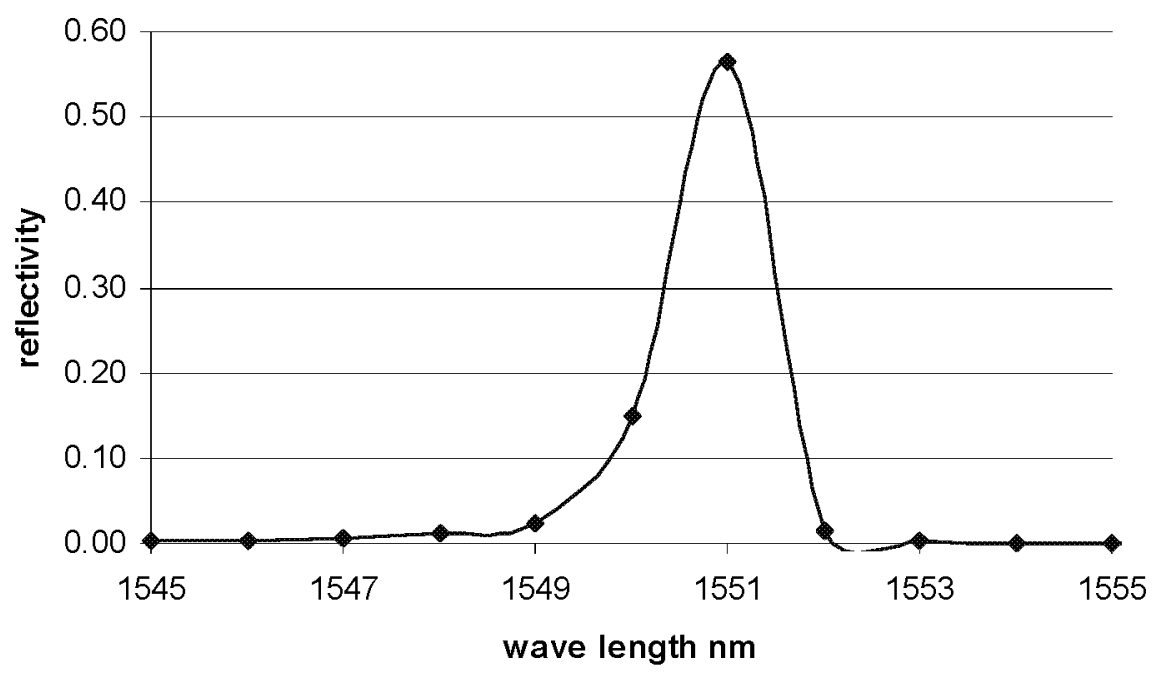

Fig. 10 strain has a random distribution where the greatest strain $=10^{-3}$ 


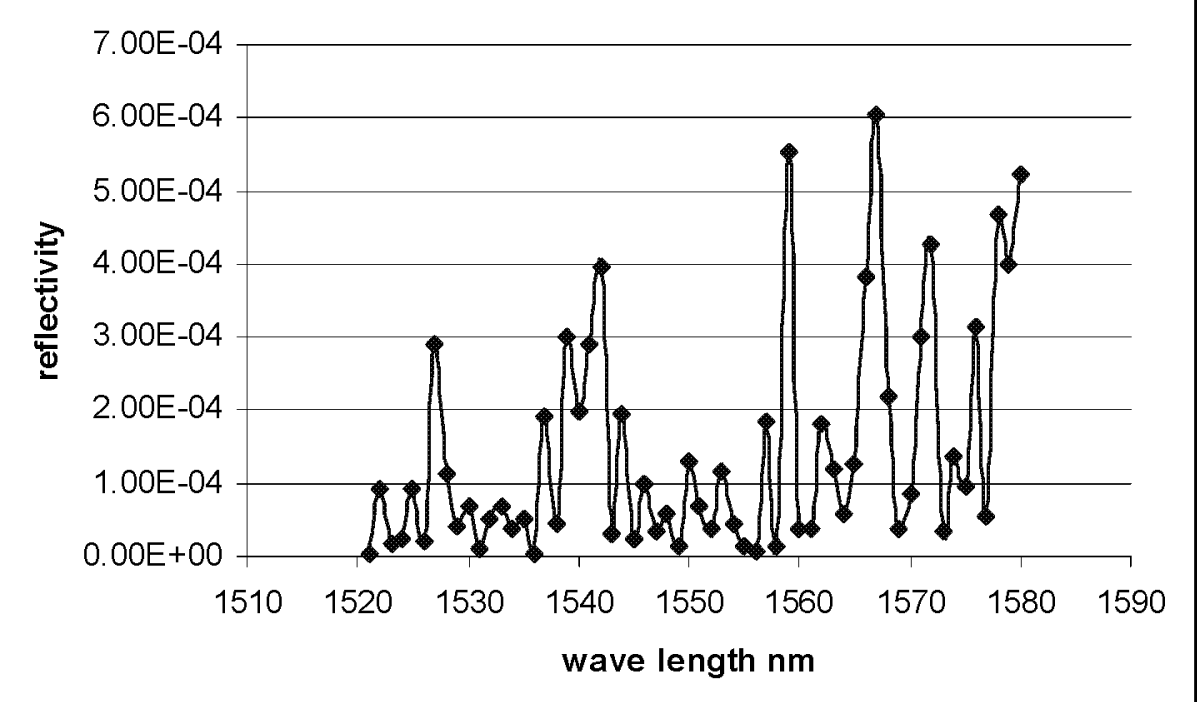

Fig. 11 strain has a random distribution where the greatest strain $=10^{-1}$

\section{MODULATION OF INDEX OF REFRACTION DUE TO STRAIN}

The applied stress to the grating affects not only the period but also the index of refraction due to photoelastic effect that may be important depending upon the magnitude of strain. The variation of index of refraction in the axial-direction for a fiber subjected to a tensile strain is given by ${ }^{20}$

$\Delta n_{p} \simeq-n^{3} p_{11} \varepsilon / 2$

where $p_{11}$ is one of the elements of the photo-elastic constant which has been measured ${ }^{21}$

and $\varepsilon$ is the local strain. We adopt the value $p_{11}=0.121$ throughout the calculation. In the following two cases are shown;

\section{4a. Uniform strain with photo-elastic effect}

We assume a uniform strain is applied to the whole fiber and therefore the Bragg grating core indices are changed to $n+\Delta n_{p}$ and $n+\Delta n+\Delta n_{p}$. Figs. 12 and 13 show the cases of $\varepsilon=10^{-3}$ and $\varepsilon=10^{-2}$ with photo-elastic effect. If we make a careful comparison between Fig. 12 and Fig. 1, we noticed the peak of reflected spectrum is shifted toward to the right (higher wavelength) by $0.25 \mathrm{~nm}$ with the photo-elastic effect considered. Compare Fig. 13 and Fig. 2 for the case $\varepsilon=10^{-2}$, we found out the peak shifts toward right by $2 \mathrm{~nm}$ which is rather significant. 


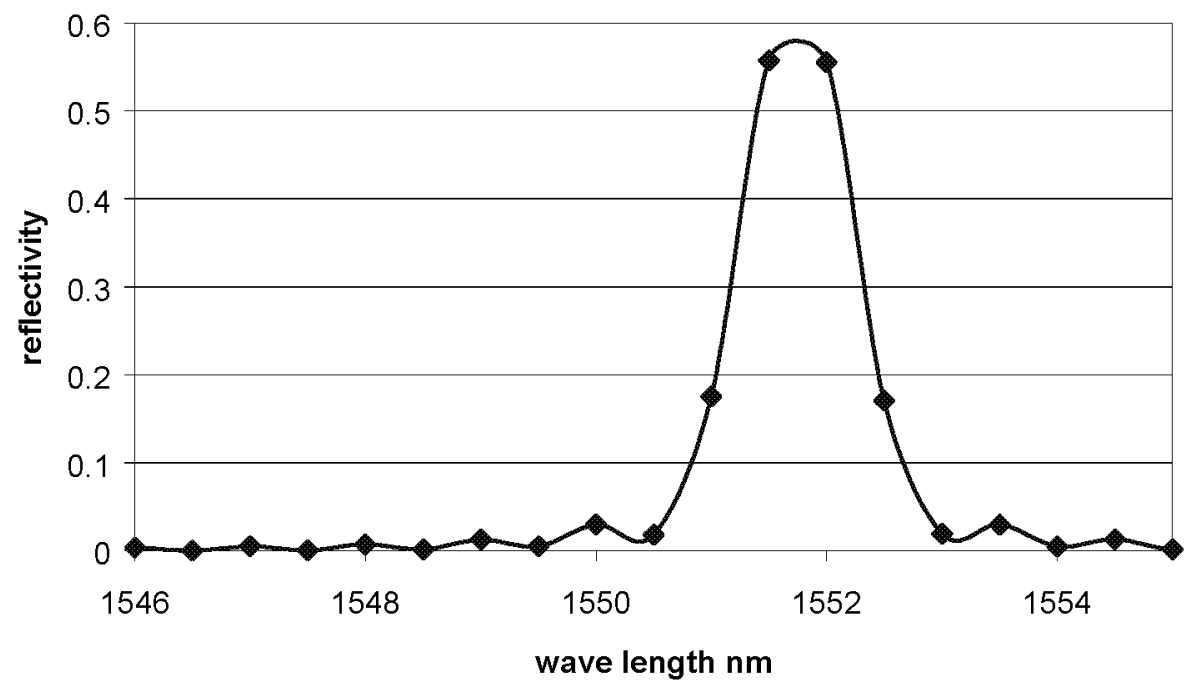

Fig. 12 uniform strain with photo-elastic effect $\varepsilon=10^{-3}$

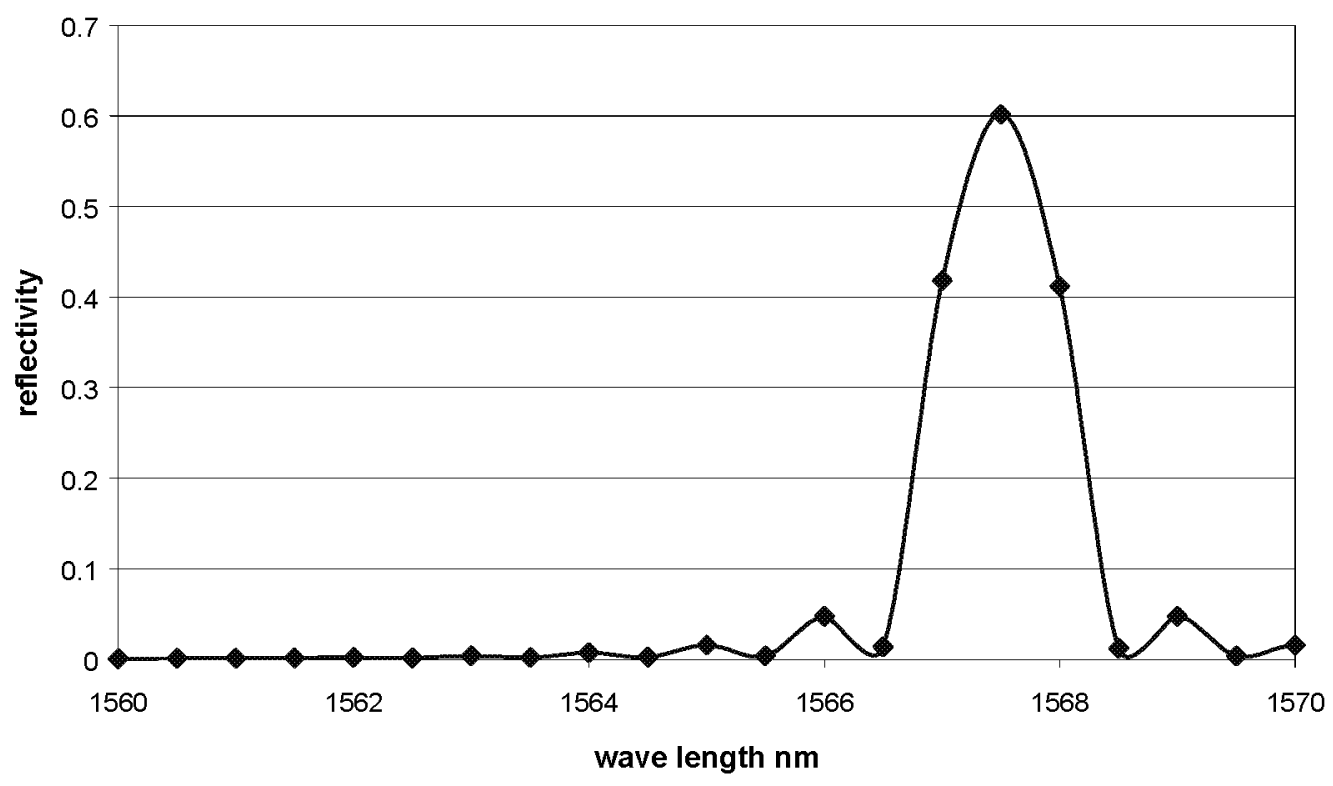

Fig. 13 uniform strain with photo-elastic effect for $\varepsilon=10^{-2}$

\section{4b. Linear strain with photo-elastic effect}

As we have done before for a linear distribution of the strain but with photo-elastic effect added on, however the magnitude of photo-elastic depends on the local strain which varies linearly. Experimentally it is hard to situate the Bragg grating sensor unless the sensor is clamped on one end where the strain is zero. Figs. 14 and 15 show the reflectivity versus wavelength. Comparing Fig. 15 and Fig. 5, the reflectivity spectra have changed greatly. 


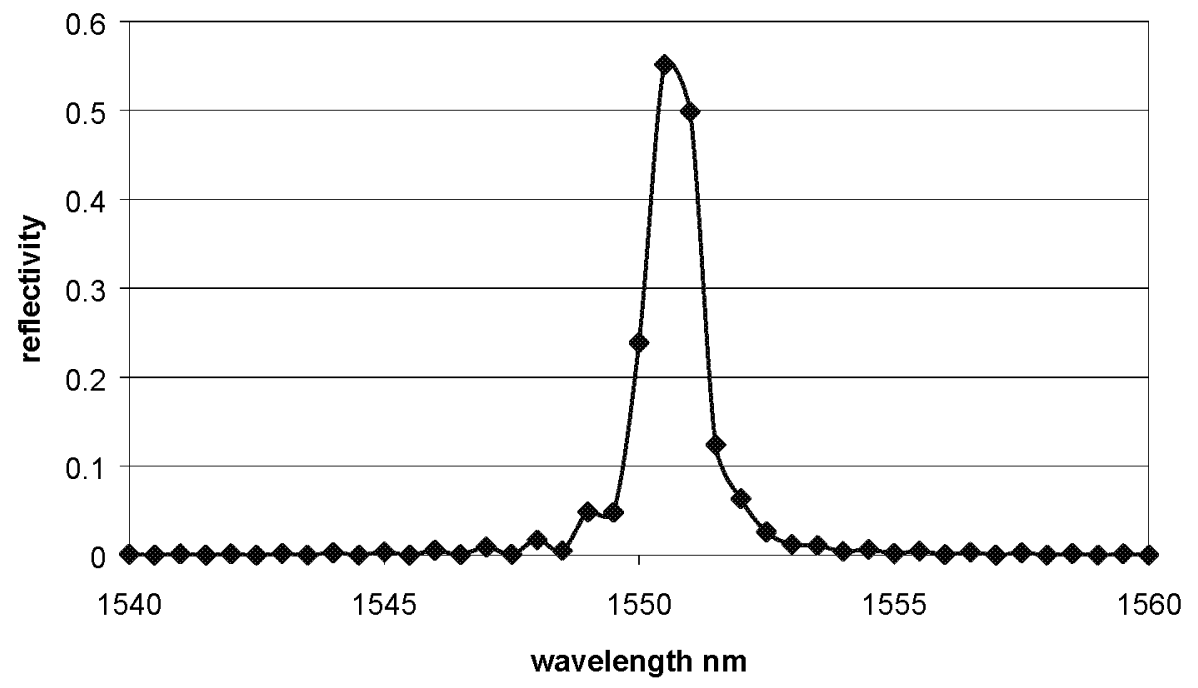

Fig. 14 linear strain with photo-elastic effect for $\varepsilon=10^{-3}$

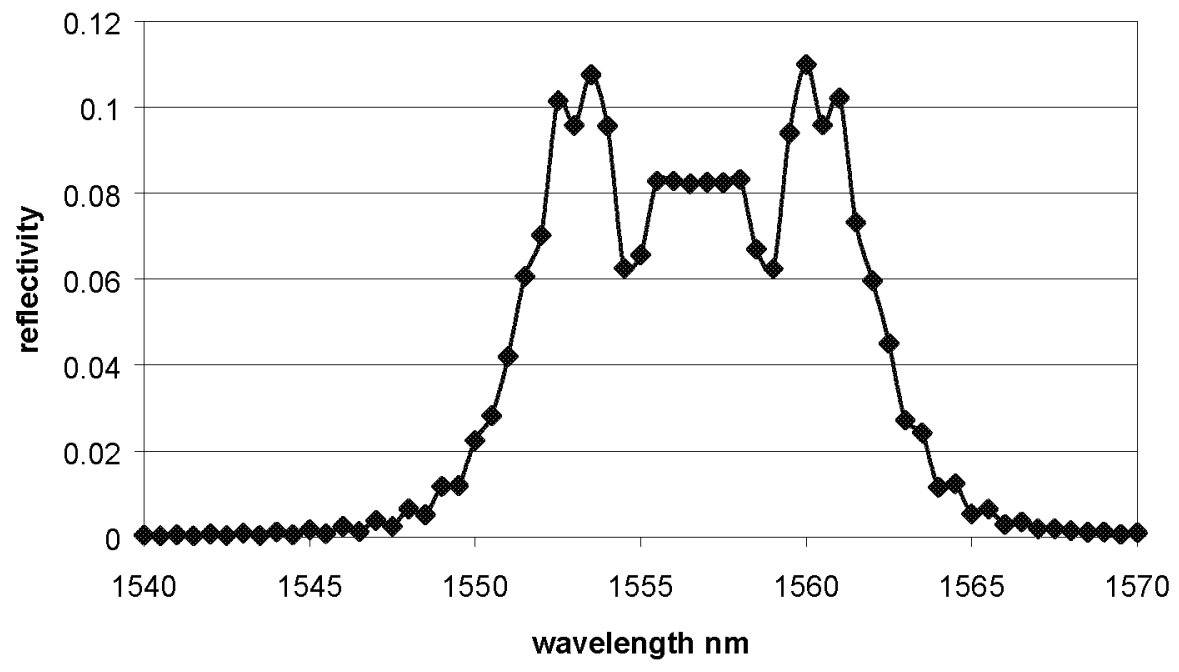

Fig. 15 linear strain with photo-elastic effect for $\varepsilon=10^{-2}$

\section{CONCLUSIONS}

Using a well established, simple plane wave matrix theory for simulating a fiber Bragg grating, we have obtained the reflected spectra for different non-uniform strain distributions. Furthermore, we have established that large non-uniform strain conditions are responsible for a multi-peaked reflection spectrum. Whereas for uniform strain, irrespective of magnitude, all reflected spectra show a unique and well defined sharp peak which can be used to infer the strain. Our simulations indicate that for non-uniform strain, the multi-peak spectra occur when the strain has reached to the order of $10^{-2}$ or greater. If the strain is small, say less than $10^{-3}$, non-uniformity is not an important issue; all the reflected spectra would give a sharp peak and uniquely determine the strain. However when the strains increase to the order of $10^{-2}$, the spectrum is broadened and splits into multiple peaks. In addition, we have demonstrated that the photoelastic effect is important when the strain is in this range also. These considerations must be taken into account when determining the strain from the maximum of the reflected spectrum. In the case of linear 
non-uniform strain the mid point of the spectrum seems to be the correct value to choose but this may not be valid for other cases. Finally, when the strains increase beyond $10^{-2}$ for a non-uniform grating, the reflected signals can be completely lost, which has been observed in some of our experiments ${ }^{22}$. This is a simple and quick numerical simulation to describe the relationship between reflected spectra and grating structures. These phenomena can be understood in a qualitative sense, i.e., each grating plane defines a reflected and selected wavelength. And when the grating distance is constant, all the reflected waves contributed constructively, creating a strong peak uniquely defining the grating distance. But when the successive grating distances are off slightly, each distance selects a slightly shifted wavelength. Therefore each back-scattered wave contributes non-coherently and the multi-peak spectra are produced. On the other hand, it is well known and has been tested here that well defined and distinguishable multi-peaked spectra can be obtained by carefully arranging a group of gratings; each group has an identical grating distance which defines the peak wavelength. The number of grating periods in each group defines the relative reflectivity strength.

\section{References}

1. Shyh Wang, J. Appl. Phys., Vol. 44, No.2, p767 1973.

2. D. K. W. Lam and B. K. Garside, Appl. Optics Vol. 20, No. 3 p440 1981.

3. Amnov Yariv, IEEE J. Quantum Electron., Vol. QE-9, No. 9, 1973.

4. D. Marcuse, B.S.T.J., Vol 52, No. 6, 1973.

5. A. W. Snyder and W. R. Young, J. Opt. Soc. Am., Vol. 68, p297, 1978.

6. C. Yeh, J. Appl. Phys. Vol. 33, No 11, p3235, 1962.

7. J. E. Sipe, L. Poladian and C. Martijn de Sterke, J. Opt. Soc. Am., Vol. 11, No. 4, p 1307, 1994.

8. David J. Griffiths and Carl A. Steinke, Am. J. Phys., Vol. 69, No. 2, p137, 2001.

9. Makoto Yamada and Kyohei Sakuda, Appl. Optics, Vol. 26, No. 16, p3474, 1987.

10. Kim A. Winick, Appl. Optics, Vol. 31, No. 6, p 757, 1992.

11. S. T. Peng, IEEE Trans. Micro. Wave and Tech. Vol. MTT-23, No. 1, p 123, 1975.

12. M. LeBlanc, S. Y. Huang, M. Ohn and R. M. Meaasres, J. Opt. Soc. Am., Vol. 21, No. 17, p1405, 1996.

13. Boo-Gyoun Kim and Elsa Garmire, , J. Opt. Soc. Am., Vol. 9, No. 1, p 132, 1992

14. Pochi Yeh, Amnon Yariv, and Chi-Shain Hong, , J. Opt. Soc. Am., Vol. 67, No. 4, p 423, 1977.

15. U. Bandelow and U. Leonhardt, Opt. Comm., Vol. 101, No. 1,2 , p 92, 1993.

16. M. Born and E. Wolf, "Principles of Optics", Pergamon Press, Oxford. 1975.

17. A. K. Ghatak and K. Thyagarajan, "Optical Electronics", Cambridge Univ. Press, 1989.

18. A. K. Ghatak and K. Thyagarajan, "Fiber Optics", Cambridge Univ. Press, 1998.

19. A. Othonos and K. Kalli,"Fiber Bragg Gratings", Artech House, Inc., 1999.

20. Zubia, J., Arrue, J., and Mendioroz, A.,Opt. Fiber Technol., 3 (2) pp. 162-167, 1997. Zubia, J., Arrue, J., IEE Proc.-Optoelectron., v.144, No. 6, Dec., 1997

21. Dixon, R.W. J. App. Phys 38, 5149-5153, 1967.

22. Robert Rogowski, private discussion. 